\title{
SUSCEPTIBILITÉ DE RATTUS NORVEGICUS ET RATTUS RATTUS FRUGIVURUS DE LA VILLE DE RECIFE À LA PASTEURELLA PESTIS
}

\author{
Dalva A. de Mello
}

\begin{abstract}
L'aùeur a vérifié la susceptibilité de deux espèces de rongeurs domestiques de la ville de Recife. Etat du Pernambuco, R. norvegicus et Rattus rattus frugivurus, les comparant aux souris albinos de la souche "Swiss" avec deux souches de P. pestis dont une était isolée au municipe d'Exu, Etat du Peinambuco cienommée PEXU 19 et l'autre provenante du Venezuela dite RANGEL.

Les deux espèces de rongeurs ont montré une resistence modérée par rapport aux deux souches de $\mathrm{P}$. pestis tandis que les souris ont révélé d'être hautement susceptibles.
\end{abstract}

\section{INTRODUCTION}

Quoique la peste au presente n'arrive pas à créer un problème sanitaire sérieux au Brésil, elle y existe quand même et en des foyers invétérés dans les zones ruraux.

On pourrait cependant croire dans un mouvement au passé ou plutôt renvoyer le cas de la peste au milieu urbain. C'est de ce fait que le Règlement Sanitaire International (1961) article 51, (3) défend la collecte sistématique des rongeurs domestiques et de ses ectoparasites pour des examens reguliers sur l'endemie ci-dessus mentionné, car il est certain que de tels animaux ont été autrefois les principaux responsables des grandes epidémies de la peste.

L'auteur considère que les données ci-dessus mentionées méritent d'être étudiées par la susceptibilité de deux espèces domestiques de grande occurrence dans la ville de Recife (Etat du Pernamibuco); en outre, il justifie encore le fait que jusqu' à présent il n'existe pas des données corrélatives à ce sujet.

\section{MATERIAUX ET METHODES}

Les travaux on été initiés au mois de Mars 1967 et terminés au mois de Novembre de la même année.

1 - RONGEURS: On a utilisé Rattus rattus frugivurus et Rattus norvegicus dans un total de 112 individus pour la première espèce et 277 pour la seconde. Les animaux ont été capturés par le secteur de Recife (Departamento Nacional de Endemias Rurais - Circunscrição Pernambuco), et mis au Laboratoire, où il était fait l'enlevement des puces et désinfectés et en suite placés dans une d'observation dans une période de deux semaines avant d'être inoculés.

Pour la comparaison des résultats à être obtenus sur les espèces ci-dessus mentionnées ont été utilisés de souris albinos de souche "Swiss" âgé de 4 mois et maintenues dans 
le biotérium du Centro de Pesquisas Aggeu Magalhães.

2 - SOUCHES UTILISEES: Deux SOUches de $P$. pestis ont été utilisées, dont une était nommée PEXU 19, isolée récemment par le $\mathrm{Dr} . \mathrm{M}$. Balmayar (Expert en peste de l'Institut Pasteur d'Iran) d'un rongeur sylvestre Oryzomys subflavus provenant du municipe de Exu, état du Pernambuco, et une autre RANGEL, offre du Dr. Bica (Organização Panamericana da Saúde), isolée en 1960 aussi d'un rongeur sylvestre (Sigmodon hispidus) en Camp. RANGEL, Venezuela.

Avant le debut du travail, les cultures ont été maintenues par des passages successives dans des cobayes dans le but de maintenir la virulence. Pour chaque expérience, les cultures isolées plusieurs fois ont été ensemencées dans l'eau peptonnée et incubées a $37^{\circ} \mathrm{C}$. Après 24 heures $d$ ' incubation elles ont été retirées de l'étuve pour le comptage des bactéries.

3-COMPTAGE DE GERMES: Le comptage de germes a été fait par l'hematimètre de Neubauer et le colorant utilisé, le bleu de Loeffler. Les cultures ont été maintenues au cours de la période des inoculations, dans une boite à glace.

4 - INOCULATIONS: La tecnique choisie pour les inoculations a été la sous-cutanée. Les rongeurs domestiques ont été distribués en groupes de 10 paires pour chaque dosage et les souris en groupes de 5 et le nombre des doses a varié de 4 à 9 pour chaque souche et pour chaque espèce d'animal. Les individus inoculés qui ne sont pas morts aux des premiers jours ont restés en observation jusqu' à le 20'me jour, moment ils ont été sacrifiés. Il était fait pour chacun bactérioscopie du foie, du poumon et de la rate au bleu de Loeffler pour so certifier de l'infection pesteuse.

5 - LE CALCUL DE LA $D L_{50}$ - a été fait d'après la méthode de LITCHFIELD \& WILCOXON (2) .

\section{RESULTATS}

Les deux espèces de rongeurs, Ratizs rattus frugivurus et Rattus norvegicus bien que les souris ont montré des différences statistiquement significatives comme susceptibilité en relation aux souches de $P$. pestis (Tableau I, II et III) .

Alors que la $\mathrm{DL}_{50}$ (Tableau I) de la souche PEXU 19 pour le Rattus rattus frugivurus est été de 9.600 (10.145-9.030), pour le Ratius norvegicus il a été de $4.700 \ldots$ $(5.080-4.300)$ dont sa relative activité ( $\mathrm{Ta}-$ bleau III) pour cette dernière espèce 2,24 à 1,8 fois plus grande que le Rattus rattus frugivurus.

La souche RANGEL en ces deux espèces de rongeurs a montré la $\mathrm{DL}_{\tilde{5} 0}$ pour le Rattus rattus frugivurus de l'ordre de.... $300.000(367.500-24.000)$ et pour le Rattus norvegicus de l'ordre de 7.800 (9.204-6.610'. La relative activité de cette souche pour le Rattus norvegicus est de 49,90 à 29,00 fois plus grande que pour le Rattus rattus frugivurus.

L'espèce $R$. norvegicus a montré plus grande susceptibilité en faveur du PEXU 19 que la RANGEL, dont sa relative activité de la première souche sur la seconde est $1,98-1,37$.

L'espèce Rattus rattus frugivurus, par rapport aux deux souches a montré plus susceptibilité en faveur du PEXU 19 que pour le RANGEL. L'activité du PEXU 19 est de $39,00-25,00$ fois plus grande que la RANGEL.

Pour les souris (Tableau II) la $\mathrm{DL}_{50}$ en fonction de la souche PEXU 19 est de 0,46 (0,50-0,42) tandis que la RANGEL a montrés la $\mathrm{DL}_{50}$ de l'ordre de $1.400(5600-350)$.

L'activité relative (Tableau III) de la souche PEXU 19 pour les souris reste entre 608 - 152 fois plus grande que la RANGEL.

Si l'on compare les résultats obtenus chez les souris aux souches PEXU 19 et RANGEL par rapport au Rattus rattus frugivurus, on peut vérifier que l'activité relative de la première souche sur ce rongeur domestique reste entre 21.840 et 19.800 et par rapport à la seconde entre 5.355 et 856,80 .

Si l'on compare encore les résultats obtenus chez les souris aux souches PEXU 19 et RANGEL par rapport au Rattus norvegicus on vérifie que l'activité relative de la souche PEXU 19 pour cet individu reste 
'TABLEAU I - SUSCEPTIBILITÉ DE RATTUS NORVEGICUS ET RATTUS RATTUS FRUGIVURUS DE LA VILLE DE KEECIFE A LA

\begin{tabular}{|c|c|c|c|c|c|c|c|c|c|}
\hline Souches & $\begin{array}{c}\text { N. } \\
\text { cilles }\end{array}$ & 750.000 & 412.500 & 75.000 & 41.250 & 7.500 & 4.125 & 750 & $\mathrm{DL}_{511}$ avec LC $95 \%$ \\
\hline \multicolumn{10}{|c|}{ RATTUS NORVEGICUS } \\
\hline PEXU 19 & & $10 / 10$ & $10 / 10$ & $7 / 10$ & $8 / 10$ & $7 / 10$ & $5 / 10$ & $0 / 10$ & $4.700(5.080-4.300)$ \\
\hline RANGEL & & $10 / 10$ & $10 / 10$ & $9 / 10$ & $6 / 10$ & $4 / 10$ & $2 / 10$ & $2 / 10$ & $7.800(9.204-6.610)$ \\
\hline
\end{tabular}

RATTUS RATTUS FRUGIVURUS

\begin{tabular}{l|c|c|c|c|c|c|c|r|r|r|r|r}
\hline PEXU 19 & $10 / 10$ & $10 / 10$ & $9 / 10$ & $4 / 10$ & $1 / 10$ & $2 / 10$ & - & $9.600(10.145-9.030)$ \\
RANGEL & $9 / 10$ & - & $4 / 10$ & - & $1 / 10$ & - & $0 / 10$ & $300.000(367.500-24.000)$ \\
\hline
\end{tabular}


TABLEAU II - SUSCEPTIBILITÉ DE SOURIS SCUCHE "SWISS" ÂGE DE 4 MOIS A LA PASTEURELLA PESTIS Mars — Novembre 967

\begin{tabular}{|c|c|c|c|c|c|c|c|c|c|c|c|}
\hline Souches & $\begin{array}{l}\mathrm{N}^{\circ} \text { de ba- } \\
\text { cilles }\end{array}$ & 7.500 .000 & 750.000 & 75.000 & 7.500 & 750 & 75 & 7,50 & 0,75 & 0,75 & $\mathrm{DL}_{50}$ avec LC $95 \%$ \\
\hline RANGEL & & $4 / 5$ & $3 / 5$ & $5 / 5$ & $1 / 5$ & $2 / 5$ & $1 / 5$ & $1 / 5$ & - & - & $1.400(5.600-350)$ \\
\hline PEXU 19 & & - & $5 / 5$ & $5 / 5$ & $4 / 5$ & $5 / 5$ & $5 / 5$ & $5 / 5$ & $2 / 3$ & $3 / 2$ & $0,46 \quad(0,50-0,42)$ \\
\hline
\end{tabular}


TABLEAU III - L'ACTIVITÉ RELATIVE AVEC LIMITES DE CONFIANCE 95\%, ENTRE SOURIS ALBINOS "SWISS", RATTUS RATTUS FRUGIVURUS ET RATTUS NORVEGICUS DE LA VILLE DE RECIFE À LA PASTEURELLA PESTIS

Mars - Novembre 1967

Relation entre les animaux

Activité relative avec LC $95 \%$

SOUCHE PEXU 19

Souris / $R$. r. frugivurus

Souris / $R$. norvegicus

$R$. $r$. frugivurus / $R$. norvegicus
$21.840-19.800$

$10.710-9.710$

$2,24-1,80$

\section{SCUCHE RANGEL}

Souris / R. r. frugivurus

Souris / $R$. norvegicus

$R$. norvegicus / $R$. r. frugivurus

$\begin{array}{cc}5.355 & -856,80 \\ 11,20 & -\quad 2,80 \\ 49,90 & -\quad 29,00\end{array}$

entre 10.710 et 9.710 , et pour la souche RANGEL entre 11,20 et 2,80 .

Dans les examens bactérioscopiques on a trouvé de baciles de la pesie en plus grosse quantité chez les souris que chez le Rattus norvegicus et le Ratius rattus frugivurus.

\section{CONCLUSIONS}

Pour le cas, deux espèces de rongeurs comestiques utilisés dans nos expérimentations la variation de la susceptibilité pour les deux souches de $P$. pestis a été très importante. En comparant les résultats obtenus chez souris et les Rattus rattus frugivurus e Rattus norvegicus ces différences sont encore plus évidentes et pas seulement par rapport à la susceptibilité d'une espèce d'individu à une souche, mais aussi susceptibilité entre les trois espèces pour chaque souche.

Quan \& coll. (4) en des travaux effectués au Hawai en des différentes zones a rencontré des résultats compactibles à ceux qui ont été obtenus ici pour le Rattus norvegicus, quoique l'auteur mentionné ait utilisé des souches de $P$. pestis différentes des nôtres
En tenant compte du concept de Holdendried \& coll. (1) par l'observation et l'analyse des tableaux I et II tant pour le Rattus norvegicus comme pour le Rattus rattus frugivurus ils peuvent être considérés comme étant moyennement résistants aux deux souches de $P$. pestis utilisés tout en étant plus grande la resistence de la dernière espèce alors que les souris peuvent être considérées susceptibles.

Les souches PEXU 19 et RANGEL d'après le concept de Sokhey (5) peuvent être considérées comme hautement virulentes pour les souris albinos de la souche "Swiss" âgé de 4 mois. Cependant, il faut dire qu'une fois fait les passages de ces deux souches en cobayes par vois percutanêe au dos, la souche RANGEL montrait toujours une réaction beaucoup plus accentuée que le PEXU 19 dont il pouvait mème entraîner la mort de l'animal ce que n'arrivait pas pour la dernière.

Par rapport aux deux espèces de rongeurs domestiques par nous utilisés les deux souches présentaient une baisse virulence, car selon Sokhey (5) une souche de $P$. pestis ne peut être considérée comme virulente qu' à condition de 6-12 organismes soient suffisants pour tuer $10 \%$ des individus inoculés. 


\section{$S U M M A R Y$}

Studies on susceptibility of domestic rodents ( $\mathrm{R}$. $\mathrm{r}$. frugivurus and $\mathrm{R}$. norvegicus) from Recife, Brazil, to two strains of Pasteurella pestis were made. The strains of $\mathrm{P}$. pestis used in the experiments were isolated from wild rodents, one (PEXU 19) in Exu, State of Pernambuco an another (RANGEL) in Venezulela. "Swiss" mice were used for comparative purposes.

Domestic rats showed to be moderatly resistant to both $\mathrm{P}$. pestis strains, while the swiss mice were considered highly susceptible to them.

\section{BIBLICGRAPHIE}

1 - HOLDENDRIED, R., and QUAN, S.F. - Susceptibility of New Mexico rodents to experimental plague. Pub. Health Rep., 71: 979-984, 1965.

2 - LITCHFIELD, J. T., and WILCOXON, F. - A simplied method of evaluating dose - effect experiment. J. Pharmocol. \& Exper. Therap., 96: 99-113, June, N. ${ }^{\circ} 2,1949$.

3 - O.M.S. - Intern. San. Regulat.
Annotated Edition, 1961, 27-31 chapter I - Plague: Article, 5i.

4 - QUAN, S.F. and coll. - The susceptibility of Ratus species from two areas of Hawail to infection with Pasteurella pestis. 4: 638-642, 1965.

5 - SOKHEY in POLITZER, R. - "Plague Studies, 3 - Problems in immunology". Bull. Org. Mond. Santé. 5: 165-226, 1952. 